\title{
III.
}

\section{Über kongenitale Lipome und schwanzähnliche Bildungen beim Menschen.}

\author{
Von \\ Friedrich Werner. \\ (Mit 1 Textabbildung.)
}

Fettgeschwülste und andere Tumoren der Kreuzsteißbeingegend sind oft beschrieben worden als schwanzähnliche Bildungen oder als Menschenschwänze, und zwar haben manche Schriftsteller sie ganzen Völkerschaften zuerkannt. Sehen wir von den fabelhaften Völkern in Indien und ihren griechischen Berichterstattern Skylax, Hekataeus, Herodot, Ktesias und Megasthenes ab, so finden wir die erste Nachricht über geschwänzte Menschen im Altertum bei Plinius im ersten Jahrhundert n. Chr. Er sowohl wie im zweiten Jahrhundert auch Ptolemaeus und Pausanias sprechen von Statyrn; Plinius und Pausanias verlegen dieses geschwänzte Volk nach Afrika, Ptolemaeus auf die Inseln des indischen Archipels. Im Mittelalter, zu Ende des 13. Jahrhunderts, berichtet Marco Polo über geschwänzte Menschen auf Sumatra. In der Neuzeit endlich entspricht die Masse der Nachrichten über das Vorkommen von geschwänzten Menschen dem Verbreitungsgebiete dieser; außer dem Zentrum, dem malaiischen Archipel, werden die Philippinen, Formosa, Japan, Rußland, Albanien, Südamerika und Afrika als von geschwänzten Menschen bewohnt angeführt. Die Völkerstämme von Inner-Afrika, besonders die Niamniam, sind dagegen aus der Reihe der geschwänzten Menschen ausgeschieden, da es sich, wie $\mathrm{Sch}$ w infurth und andere Forscher feststellten, bei ihnen nicht um ein Geschwänztsein, sondern um künstliche, sogenannte Kostümschwänze handelt. M 0 h n i k e, dem wir in seinem Buche: „Über geschwänzte Menschen, Münster 1878“, die erste Zusammenstellung der Nachrichten über geschwänzte Menschen verdanken, glaubt auch alle übrigen von ihm berichteten Fälle teils wegen Unglaubwürdigkeit, teils wegen Leichtgläubigkeit der Berichterstatter als nicht einwandsfrei hinstellen zu können. Ferner hat es sich Bartels in seiner Abhandlung: ,Über Menschenschwänze" im Archiv für Anthropologie, 


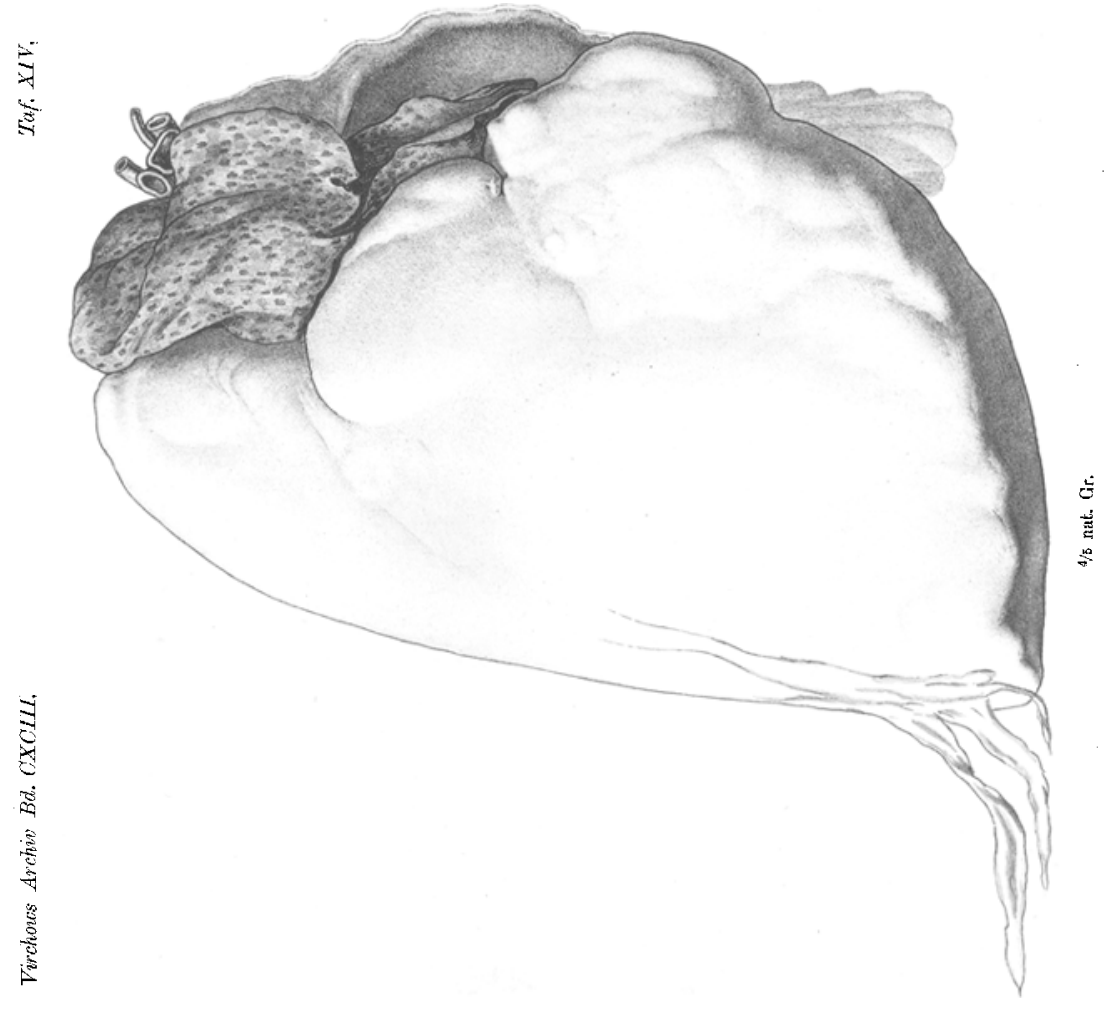


XIII. Bd. S. 1-41, angelegen sein lassen, alle in der Geschichte vorkommenden und nach $\mathrm{Mohnike} \mathrm{noch} \mathrm{bekannt} \mathrm{gewordenen}$ europäischen Fälle von Schwanzbildungen zusammenzustellen und nachzuprüfen. Er kommt zu dem Ergebnis, „daß bis jetzt eine Schwanzbildung beim Menschen, welche durch Atavismus ihre Erklärung finden könnte, in unanfechtbarer Weise noch nicht beobachtet worden ist" ( $\mathrm{B}$ artels a.a. O. S. 30).

Virch ow gibt in der Berliner klinischen Wochenschrift, Jahrgang 1884, Nr. 47 S. 746, folgende Erklärung über einen wahren Menschenschwanz: „Die weichen Schwänze sind ja unzweifelhaft alle keine wahren Schwänze, denn ein wahrer Schwanz an einem Wirbeltier setzt eben auch eine Verlängerung der Wirbelsäule mit Vermehrung der Wirbelkörper innerhalb des Schwanzes selbst voraus; er kann nicht ein bloßer weicher Strang sein. Aber wenn dieser weiche Strang aus einer ursprünglich spinalen oder vertebralen Anlage hervorgegangen ist und nur ein Rudiment einer früher vorhanden gewesenen Verlängerung dieser Anlage repräsentiert, so wird man ihn ebensowenig einen falschen Schwanz nennen können. Es ist ein nicht zur vollkommenen Ausbildung gelangter, aber doch immerhin ein Schwanz. Man könnte sich daher, wie mir scheint, vorläufig damit aushelfen, daß man diese Schwänze unvollkommene Schwänze nennt, Caudae.imperfectae."

Am meisten haben wohl Tumoren, die mit Spina bifida occulta vergesellschaftet waren, in früheren Zeiten die sogenannten Menschenschwänze vorgetäuscht, weniger die Lipome und Fibrome, denn sie scheinen sehr selten oder zum wenigsten doch sehr selten der Erwähnung werte Tumoren gewesen zu sein. - B r a u n e sagt in seinem Buche: „Die Doppelbildungen und angeborenen Geschwülste der Kreuzbeingegend in anatomischer und klinischer Beziehung“, Leipzig 1862, S. 75, von denselben: „Wenn auch die angeborenen Lipome nicht so selten sind als $\mathrm{L}$ o t $\mathrm{zbeck}$ meint, der kein Beispiel davon aufinden konnte, so sind doch die sicheren hicrher gehörigen Beobachtungen so spärlich, daß, wenn man nicht die sogenannten Schwanzbildungen ohne knöcherne Grundlage mit dazu rechnen würde, nur die L a n g e n b e c k schen Beobachtungen übrig bleiben dürften. Ich habe es daher für zweckmäßig gehalten, die Lipome mit den Schwanzbildungen zusammen abzuhandeln." Er führt die folgenden sechs Fälle an: 
,M i d d e l d o r f f. Angeborenes Lipom der Steißbeingegend. Abtragung mit der glühenden Schneideschlinge. Heilung. Herr Prof. M i d d e ld o r f f hatte die Güte, mir brieflich die folgende Mitteilung zu geben über den Fall, der von L o t z be ck a. a. O. S. 59 und $\mathrm{Zs} \mathrm{igmondy,} \mathrm{Wiener} \mathrm{medizinische} \mathrm{Wochenschrift} \mathrm{1858,}$ nur kurz erwähnt ist.

Am 6. August 1857 wurde der elfmonatliche Sohn des Stellmachers Friedrich Schur in die Breslauer Universitätspoliklinik gebracht. Er hatte einen angeborenen daumendicken, birnförmigen, dünngestielten Anhang am Schwanzbeine, von runzliger, glänzender, fettiger Haut überzogen. Der Tumor bestand, wie sich nachher ergab, aus fettreichem Bindegewebe und wurde mit der galvanokaustischen Schneideschlinge ohne Blutung und mit glücklichem Erfolge abgetragen.

$\mathrm{L}$ a n g e $\mathrm{n}$ b e ck. Drei Fälle von angeborenen Lipomen der Kreuzbeingegend, die mit Erfolg exstirpiert wurden.-Deutsche Klinik 1850, S. 26. Die Bestätigung des guten Erfolges, der aus der kurzen Mitteilung schon erwartet werden konnte, habe ich von Herrn Geheimrat $\mathbf{L}$ a $\mathbf{n} \mathbf{g}$ e $\mathbf{n}$ b e c k selbst erhalten.

Der erste Fall betrifft ein halbjähriges Kind, das durch die Exstirpation von der Sakralgeschwulst befreit wurde.

Der zweite ein anderthalbjähriges, welches die Geschwulst, welche sich in die Incisura ischiad. hinein erstreckte, an der linken Beckenhälfte trug; die Exstirpation hatte guten Erfolg.

Der dritte Fall betraf ein Lipom, welches auf der Mitte des Kreuzbeins aufsaß.

Der erste Fall wurde in Berlin operiert, die beiden letzteren in Kiel, wo auch von L. ein angeborenes Lipom am Rücken eines 17 jährigen Mädchens mit Erfolg entfernt wurde.

Cantor. Angeborenes Lipom der Steißbeingegend. Exstirpation. Heilung. Lancet. 27. Oktober 1860, S. 411.

Das mit dem Lipom, welches als ,fleischige, schwanzartige Verlängerung" erschien, behaftete Kind wurde in das Charing-cross Hospital zu London gebracht. Das abnorme Gewächs bestand aus einer Fortsetzung der Haut in der Anuskerbe, erstreckte sich nach vorn und hatte auf der einen Seite die etwas verschobene Mastdarmöffnung, ohne daß die Defäkation wesentlich behindert wurde. Die Masse wurde mit dem Messer entfernt. 
F a b e r. Angeborenes Lipom (?). Duor. monstror. hum. descript. anatom. Berolini, 1827, p. 17.

Faultoter, weiblicher Fötus, wegen Wehenschwäche extrahiert, 7 Zoll lang, mit einem über kopfgroßen, bis an die Wade reichenden Perinäaltumor behaftet, der Fettmassen und fibröse Stränge enthielt. Die Geschwulstmasse erstreckt sich bis ans kleine Becken, welches so vollständig davon erfüllt wird, daß das Bauchfell mit Darm und Blase wie in die Höhe gehoben erscheint.

(Der Verf. gibt zwar an, daß beim Einschneiden aus dem Tumor lipomatöse Masse herausgekommen sei, ähnlich dem Fettgewebe an Arm und Beinen, doch fehlen histologische Angaben; die Abbildung erinnert mehr an einen Steißbeintumor der ersten Reihe. ${ }^{66}$

$\mathrm{Zu}$ diesen sechs Fällen wäre noch der folgende zu rechnen, welchen $\mathrm{Br}$ a u n e unter „B. Schwanzbildungen ohne knöcherne Grundlage und Lipome" S. 76 erwähnt: ,R 010 f f, Mém. de l'acad. des sc. de Berlin, 1761, p. 73, ref. Me c ke l, Path. Anat. I, S. 386.-R. sah außer andern Mißbildungen an einem reifen weiblichen Fötus einen 1/2 Zoll langen Schwanz ohne Knochen." R o l off "Courte description d'un monstre humain (traduit du latin, lu le 19 de Nov. 1761). Histoire de l'Académie royale des sciences et belles-lettres. Année 1761, Berlin 1768, p. 73-80, 2 Tafeln": betrachtet diesen Tumor als Hautdivertikel. „A la partie postérieure du lombe droit, pas loin de l'os sacrum, se trouvoit attachée une pièce particulière, qui avoit l'air d'une petite queue, et pendoit de l'os sacrum, de la longueur d'un pouce et au delà. Cette queue étoit située à la surface extérieure de l'os des iles, mais seulement à la peau extérieure qui le couvre, d'où ello pendoit d'une manière lâche; sa largeur étoit égale partout, quoiqu'un peu plus grande par enhaut, et moindre par embas, mais de façon qu'elle se terminoit en un angle plutôt obtus qu'aigu. D'ailleurs cette petite queue étoit de pure chair, sans rien d'osseux ni de cartilagineux ou de musculeux. Enfin, à l'égard de cette queue quo portait le foetus à la région de l'os sacrum, on ne doit la regarder comme une simple production de la peau externe."-Außerdem hat B a r t e l s in diesem Archiv Bd. 83 S. 189 ein Lipoma fibrosum cutis coceygealis pendulum unter dem Titel ,Eine schwanzähnliche Neubildung beim Menschen" veröffentlicht. Der Fall ist folgender: 
„Als der (50 jährige) Patient sich entsprechend entkleidet und zur besseren Besichtigung der Teile ein wenig vornübergebeugt hatte, präsentierte sich zwischen den Hinterbacken ein rundlich-länglicher Körper von der Größe einer blauen Herbstpflaume, welcher mit livide rötlicher Haut überzogen das Bild eines sehr großen Hämorrhoidalknotens darbot. Wenn man die Hinterbacken aber auseinanderzog, veränderte sich das Bild in für mich wahrhaft überraschender Weise. Ich konnte mich jetzt nämlich überzeugen, daß das Gebilde gar nicht mit dem After oder dessen Rande in Verbindung stand, sondern frei über ihm hing. Es wurde von einem schmalen, häutigen Stiele getragen, welcher in der Medianlinie des Körpers am obersten Ende der Crena clunium genau an derjenigen Stelle seinen Ursprung nimmt, unter welcher die Spitze des Steißbeins liegt." B artels bemerkt u. a. hierzu: ,Sollte das Ding für einen Schwanz gelten können, so war es selbstverständlich erforderlich, daß es dem Besitzer angeboren war. Diese Anforderung wurde nun aber nach Aussage des Patienten nicht erfüllt. Sollen wir dem vorliegenden Neoplasma in dem Systeme der V i r c h 0 w schen (Geschwülste Bd. I) Nomenklatur die ihm gebührende Stelle anweisen, so würden wir wohl den Namen Lipoma fibrosum cutis coccygealis pendulum zu wählen haben."

Einen diagnostisch sehr interessanten Fall von Hygroma cysticum glutaeale congenitum teilt $R$ udolf $V$ irchow in diesem Archiv Bd. 100 S. 571 ff. mit. Es heißt dort aus dem Briefe des Herrn Stabsarztes Dr. L udwig Wolf aus Malonge an V i r c h 0 w über die exstirpierte Geschwulst: „In der ersten Kiste befindet sich in Alkohol noch ein Lipom, das ich am 3. Mai bei einem am 25. April d. J. (1884) 9 Uhr morgens geborenen Negerkinde operativ entfernte. Ich sah das Kind, weiblich, am Geburtstage, 5 Uhr nachmittags: Hautfarbe im ganzen dieselbe wie bei einem Kinde kaukasischer Rasse, nur der Rücken und das untere Drittel der Unterschenkel hoben sich etwas dunkler ab. Die Iris war braun. An der linken Gesäßbacke, in der Horizontallinie etwa $2 \mathrm{~cm}$ von der Afteröffnung, befand sich ein stielförmiger Tumor, $9 \mathrm{~cm}$ lang und $15,3 \mathrm{~cm}$ im Umfang (breit). Die Körperlänge des Kindes betrug $52 \mathrm{~cm}$. Die Eltern wünschten die operative Entfernung, welche dann auch von mir vorgenommen wurde. Starke arterielle Blutung, Unterbindung, Karbolverband, Heilung per 
primam int. Die unmittelbar vor der Operation gemachte Messung ergab, daß der Trmor gewachsen war. Die Länge betrug $9,5 \mathrm{~cm}$, die Breite 15,8cm." - Die Untersuchung der Geschwulst von V i r c h ow ergab: „Die Geschwulst bot äußerlich in der Tat ganz das Aussehen eines gestielten Lipoms dar, und Herr Dr. L. W o I f hatte um so mehr Grund, sie für ein solches zu halten und demnach nur mit der Haut und Unterhaut in Beziehung zu setzen, als die Ansatzstelle des engen Stiels an der linken Seite des Gesäßes, ziemlich tief nach unten, auf irgendwelchen Zusammenhang mit tieferen Teilen, namentlich mit der Wirbelsäule in keiner Weise hindeutete. Indes ein Durchschnitt der Geschwulst zeigt einen sehr zusammengesetzten Bau und ist meiner Auffassung nach ohne Annahme eines Zusammenhanges mit der Wirbelsäule nicht verständlich." Die mikroskopische Untersuchung ergab quergestreifte Muskelbündel, Bündel junger Nervenfasern und Gefäße. Vi r c h ow schließt mit den Worten: ,Schließlich will ich darauf hinweisen, daß Fälle, wie der vorliegende sehr verlockend für diejenigen sind, welche die Frage der menschlichen Schwänze verfolgen. Man könnte dem kleinen Negermädchen in der Tat einen ,Fettschwanz" aufhängen. Indes will ich besonders hervorheben, daß ich nirgends darin Knorpel oder Knochen fand, nicht einmal, wie in dem Straßburger Präparat, einen faserknorpeligen Zentralstrang. Obwohl ich daher eine gewisse Analogie mit Schwänzen zugestehen kann, so kann von einer wahren und eigentlichen Schwanzbildung doch keine Rede sein."

Schließlich sei hier noch der von S t o l p e r in der Deutschen Zeitschrift für Chirurgie 50. Bd., 3. und 4. Heft, in seiner Abhandlung „Über die angeborenen Geschwülste der Kreuzsteißbeingegend" wiedergegebene Fall angeführt.

„Nun habe ich in Königshütte ein kaudales Anhängsel seinerzeit operiert, das ich anfänglich als einen angeborenen echten Sakraltumor auffassen zu müssen glaubte. Die nachträgliche histologische Untersuchung bestätigte indes diese Vermutung nicht. Sie ließ mich bezüglich der Herkunft der Geschwulst überhaupt keine stichhaltige Erklärung finden, und da überdies es nicht erwiesen ist, ob sie wirklich angeboren, $d$. h. schon bei der Geburt vorhanden gewesen ist, so entfällt sie eigentlich nicht in dieses Thema. Aber als Beitrag zur Differentialdiagnose dürfte der Fall doch erwähnenswert sein. 
Großes Fibrom, ausgehend von der Vorderfläche des Kreuzsteißbeines einer 42 jährigen Frau, vielleicht angeboren. Erfolgreiche Exstirpation.

Die 42 Jahre alte Bäuerin Florentine Ch. aus 0. kam am 15. Februar 1897 in unsere Poliklinik wegen einer von der rechten Gesäßhälfte herabhängenden großen Geschwulst. Dieselbe gab an, daß sie seit etwa vier Jahren die Entwicklung derselben wahrnehme, nachdem sie schon als Kind manchmal einen hühnereigroßen Knollen an dieser Stelle bemerkt habe. Seit einem halben Jahre wachse dieselbe stetig und sei ihr beim Sitzen und Gehen durch das Hin- und Herpendeln so unbequem, daß sie dieselbe gern entfernthaben möchte.

Die zwar nicht besonders kräftige, aber normal gewachsene und an den inneren Organen gesunde Frau, Mutter von vier lebenden und zwei verstorbenen Kindern, hat einen reichlich dreifaustgroßen Tumor an der Gesäßgegend, welcher in seiner Form an ein Serotum bovis erinnert. Er hängt an der rechten Hinterbacke herab und hat durch sein Eigengewicht deren Konturen erheblich verzerrt. Man sieht äußerlich eine halsartige Einschnürung zwischen der Glutäalrundung und dem Tumor, der bei jeder Bewegung hinund herpendelt. Die darüberziehénde Haut ist im allgemeinen gesund, und wo der Tumor dem linken Oberschenkel sich anlegt, besteht, offenbar infolge von Beschmutzung mit Kot und Urin, eine leichte, entzündliche Rötung. Für die Palpation ist die Haut überall leicht verschieblich um den sehr harten Inhalt. Die ganze Geschwulst läßt sich leicht nach oben schlagen und dem Kreuzbein anlagern. Die feste, eigentliche Geschwulst, über der sich die Haut offenbar nur ausgedehnt hat, steht durch einen leicht eingeschnürten Stiel, wie es scheint, mit dem Steißbein in Verbindung. Dieser Umstand sprach gegen die Vermutung, daß es sich um ein Lipom handle; ein cystischer Sakraltumor, wie sie angeboren vorkommen, war auch nicht anzunehmen; da man Cysten keinesfalls wahrneḥmen konnte.

Am 16. Februar 1897 versuchte ich zunächst unter S c h l e i c hscher Lokalanästhesie den Tumor zu exstirpieren. Da sich aber die Frau sehr ungebärdig zeigte, so wurde Chloroformnarkose alsbald in Anwendung gebracht.

Der Stiel wurde durch einen vorderen und einen hinteren Schnitt durch die Haut freigelegt. Hierbei blutete es aus den Venen 
ziemlich. stark. Als ich den Tumor nach oben hin freimachte, zeigte sich, daß der feste Stiel ziemlich weit hinaufreichte, nämlich bis an die vordere konkave Fläche des Steißbeines und des Kreuzbeines. Er ließ sich aber nach sorgfältiger Isolierung stumpf von der Kreuzbeinvorderfläche ablösen. Der Tumor hatte hier dem Periost aufgesessen. Die Auslösung in der Tiefe war wegen der aus den Sakrallöchern austretenden Nerven immerhin nicht ohne Bedenken, doch zeigte sich nachträglich, daß wir jede Nervenläsion vermieden hatten.

Die überreichliche Haut ließ einen vollständigen Verschluß der Operationswunde zu, doch wurde mit Rücksicht auf die Nachbarschaft des Afters und die vermeintliche Quetschung der Muskulatur ausgiebig drainiert. Innerhalb von etwa drei Wochen heilte die Wunde ohne erhebliche Absonderung, und die Patientin konnte bei der Entlassung jede Bewegung ohne Schmerzen oder Unbequemlichkeit ausführen. Bis jetzt - nach mehr als einem Jahre ist ein Rezidiv nicht aufgetreten. Die Narbe ist reizlos und macht sich in keiner Weise bemerkbar.

Pathologisch-anatomische Untersuchung: Die Geschwulst schneidet sich sehr schwer und erweist sich für das bloße Auge durchweg solid, die weißglänzende Schnittläche gleicht genau derjenigen von Fibromyomen des Uterus, und wie in diesen setzen sich einzelne Abteilungen des Tumors durch verschiedenen, oft konzentrischen Verlauf der Faserzüge kugelartig gegeneinander ab. Die Oberhaut ist von dem eigentlichen Tumor durch ein lockeres, in den untersten Teilen etwas ödematöses Zwischengewebe getrennt. Der Stiel, mit dem die Geschwulst der ventralen Fläche des Kreuzsteißbeines angeheftet war, ist ebenfalls von fibrösem Aussehen.

Die mikroskopische Untersuchung bestätigt durchaus die makroskopische Diagnose auf Fibrom. Es ist eine durchweg gleichmäßige Faserstruktur, die nur in den abhängenden Partieen etwas. reichlicher saftdurchtränkt ist. Die Kerne sind verhältnismäßig spärlich, länglich, nehmen überall gleichmäßig die Farbe an. An arteriellen Gefäßen ist die Geschwulst ziemlich reich, nirgends sind Zeichen einer regressiven Metamorphose zu finden. Bezüglich ihres Ausgangsortes gibt die histologische Untersuchung keinen Anhalt, nach dem Befund bei der Operation müßte man das Periost 
der vorderen Kreuzsteißbeinfläche als den Boden ansehen, auf dem die Geschwulst gewachsen ist." -

Es möge nun noch der von Herrn Professor Dr. K a r e w s k i mir gütigst überlassene Fall eines kongenitalen Lipofibroms der Kreuzsteißbeingegend mitgeteilt werden.

Am 3. Juli 1907 brachte Frau H. geb. P. in die ÄuRere Poliklinik des Krankenhauses der jüdischen Gemeinde zu Berlin ihr Kind, um ihm den gleich zu beschreibenden Tumor entfernen zu lassen.

Anamnese: Therese H. wurde am 25. Juli 1905 geboren. Sie soll im Juni 1906 einige Tage an Brechdurchfall gelitten haben. Sie länft seit ${ }^{3} / 4$ Jahren. Seitdem bemerlte die Mutter, daB das Kind krumme Beine bekam; In der Familie sollen nie Mißbildungen vorgekommen sein. Bei dem Großvater männlicherseits, der mit 64 Jahren in Dalldorf gestorben ist, traten mit 40 Jahren Krämpfe auf von $1 / 2$ stündiger Dauer, mit Bewußtseinslosigkeit und Schaum vor dem Munde. Die Krämpfe stellten sich nur nach alkoholischen Excessen ein. Sieben Monate vor seinem Tode soll er plötzlich geisteskrank geworden sein. Er erkannte seine Frau bis zuletzt. Die Krämpfe sollen nach Auftreten der Geisteskrankheit fortgeblieben sein. Während der Krankheit soll er auch getobt haben.

Eine 24 jährige Tante männlicherseits hat angeblich nach der lmpfung im Alter von einem Jahr und einem Monat Krämpfe bekommen, welche alle paar Tage, und zwar mehrmals hintereinander, auftraten. Bis zum siebenten Jahre war sie bei der Mutter, dann kam sie nach Dalldorf und später nach Wallgarten bei Biestorf. Die Krämpfe sind unverändert geblieben. Geistig soll sie auf der Stufe eines zwölfjährigen Mädchens stehen.

Eine GroBtante mütterlicherseits ist taubstumm. Diese fängt zu toben an, wenn ihre Wünsche nicht gleich erfüllt werden. Sonst ist sie friedlich und ruhig.

Die Mutter des Kindes, Frau H. geb. P., ist 20 Jahre alt. Sie will als Kind Windpocken und Masern gehabt haben und sonst immer gesund gewesen sein. Die erste Menstruation mit 131/2 Jahren. Menses drei bis vier Tage Daner, nicht sehr stark, alle vier Wochen, ohne jegliche Schmerzen. Das Kind, erster Partus, wurde zur rechten Zeit geboren. Wegen Wehenschwäche wurde es vom Arzte mit der Zange geholt. Das Kind soll gegen 4 Pfund (?) gewogen haben. Es war sehr zart gebant und soll erst nach wiederholten Atmungsversuchen zu sich gekommen sein. An der rechten Hinterbacke fand man die unten beschriebene Geschwulst: Als die Mutter im fünften Monat schwanger war, sah sie bei Bekannten zwei Affen. Der eine derselben rannte unter den Stuhl, auf dem die Mutter saß. Sie, erschrak dabej, weil sie glaubte, der Affe wollte ihr unter die Röcke kriechen, um sie zu beißen. Sie fuhr in die Höhe und umfaßte mit beiden Händen die beiden Glutäalgegenden. Früher war ihr von Bekannten erzählt worden, daß man sich während der Schwangerschaft nicht erschrecken dürfe. Als sie und ihr Mann von den Bekannten fortgingen, sagte sie zu ihm: „Siehst Du, jetzt habe ich mich doch erschrocken." Seit dieser Zeit wähnte sie, daß sie einen Affen gebären müsse. 
Status praesens: Das Kind befindet sich in gutem Ernährungszustand. Die Epiphysen der Vorderarmknochen sind etwas verdickt. Es besteht leichtes Genu varum beiderseits. Die körperliche und geistige Entwicklung des Kindes entspricht seinem Alter. Die Geschwulst wurde gleich nach der Geburt bemerkt. Sie ist von vornherein ziemlich groß gewesen und ist auch mit dem Kinde etwas gewachsen, aber verhältnismäßig wenig. Man erkennt an der rechten Hinterbacke ganz nahe der Analspalte einen Tumor, dessen unteres Ende in der Querlinie der hinteren Afterkommissur sitzt und dessen obere Begrenzung bis zur Articulatio sacrococcygea geht, sich streng halbseitig hält, so dab er senkrecht zur Raphe abfällt. Man kann an ihm zwei gut voneinander geschiedene Teile erkennen. Nämlich einen basalen, halbovoiden von der Größe eines längsdurchschnittenen Hühnereies, und auf ihm sitzend einen kleinen, kirschengroßen, schwanzförmigen, dessen Verbindung mit dem ersteren eine Einschnürung zeigt,

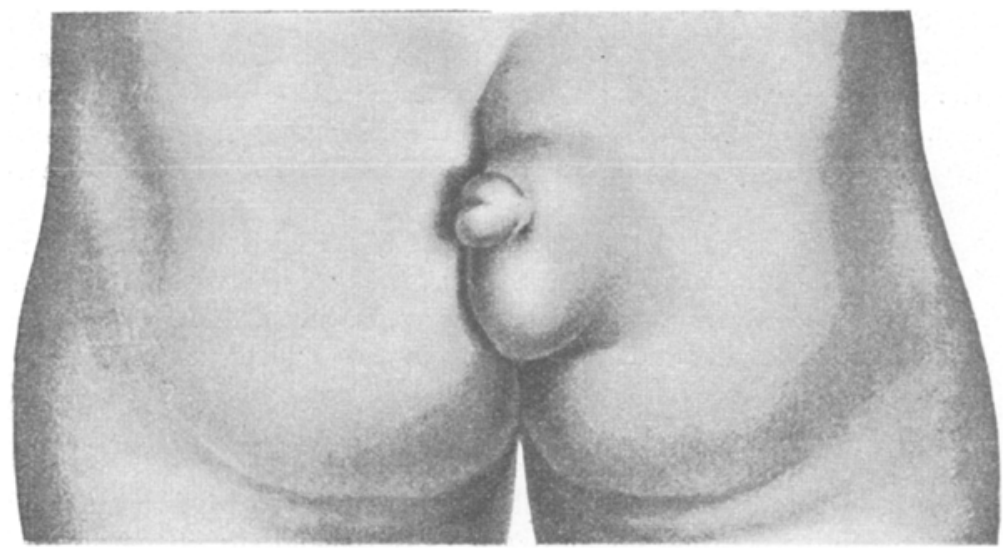

so daß er stielartig mit ihm verbunden ist. Der basale Tumor ist von einer glatten, gesunden, wenn anch etwas hyperämischen Haut bedeckt. Der peripherische hat gleichfalls normale Haut, aber diese ist auffallend stark behaart mit feinen, blonden Härchen, welche bis $2 \mathrm{~cm}$ lang sind und deren Farbe mit der des Haupthaares übereinstimmt. Quer über das äußere Ende des zweiten Tumors verläuft eine horizontale, ziemlich tiefe Furche. Unterhalb der oben beschriebenen stielförmigen Verbindung beider Geschwülste sieht man ein Loch, welches in einen $1 / 2 \mathrm{~cm}$ langen Blindsack führt, und in dem sich eine komedoähnliche Masse befindet. Die Fossa sacrococcygea ist sehr wenig ausgesprochen. Eine Fistula sacrococygea ist nicht vorhanden. Der obere Teil der Raphe zeigt eine durch den Tumor bedingte, links konvexe Ausbiegung, so da $\beta$ sie anstatt gerade bogenförmig verläuft. Dem Gefïhl nach ist die ganze Geschwulstbildung weich, lappig wie ein Lipom. Das Kind fühlt überall die ihm in die Haut des wumors applizierten Nadelstiche und macht bei solchen Abwehrbewegungen mit den Beinen, Irgendwelche Eigenbewegung ist an der Geschwulst nicht 
bemerkbar. Die ganze Bildung sieht aus wie ein Schwänzchen, das auf einer Vorwölbung der rechten Glutäalgegend sitzt. Kneift man das Kind in die Posteriora, so kontrahieren sich die Glutäen, und es erfolgt eine dieser Kontraktion entsprechende Lageveränderung der Geschwulst. Am übrigen Körper sind keine weiteren kongenitalen MiBbildungen.

Die Geschwulst wurde an der Basis ovalär umschnitten und bis in die Tiefe zur Glutäalmuskulatur verfolgt. Der basale Tumor besteht ausschlieBlich aus Fettgewebe, welches sich in keiner Weise von dem subkutanen Glutäalfett unterscheidet, noch sich von ihm abgrenzen läßt, so daß man gezwungen ist, einfach keilförmig das. Aftergebilde aus der Glutäalgegend heranszuschneiden. Man findet in der Tiefe eine Arterie und zwei Venen, welche aus dem Fett senkrecht aufwärts zu dem Schwänzchen hinziehen.

Pathologisch-anatomische Untersuchung: Die entfernte Geschwulst hat abgesehen von dem herabhängenden Teile die Größe einer Walnuß. Das Schwänzchen erscheint jetzt hammerförmig, und zwar so, daß der Stiel etwa $2 \mathrm{~cm}$, der Hammerkopf etwa $1 \mathrm{~cm}$ lang ist. Die früher beschriebene feine Öffiung ist die Ausmündungsstelle eines mit normaler Haut bekleideten engen Ganges, an dessen blindem Ende zwei schwarze Härchen gefunden wurden. Auf dem Durchschnitt erscheint die Geschwulst aus einem grobkörnigen, festen, mit fibrösen Fasern und Scheidewänden durchzogenen Fettgewebe zusammengesetzt. Nach dem Schwanz zu nimmt das Bindegewebe überhand, und dieser ist selbst so fettarm, daB er rein fibrös zu sein scheint. Die Arterie und die Venen lassen sich bis zum Schwanz hinein verfolgen. Die mikroskopische Untersuchung bestätigte ganz die makroskopische Diagnose des basalen Tumors auf Lipom und des peripherischen Tumors auf Fibrom.

Was nun im vorliegenden Fall das Versehen der Mutter des Kindes betrifft, so kann man wohl annehmen, daß durch Schreck Uteruskontraktionen angeregt werden können, die gelegentlich auf den Ablauf der Schwangerschaft von Einfluß sein mögen. Die an den Schreck anschließenden Vorstellungen der Mutter jedoch kann man ganz außer acht lassen; denn erstens war die Mutter im 5. Monat sehwanger, zu einer Zeit, wo der Embryo sehon sehr weit entwickelt war; und zweitens müßte doch, falls die unwillkürliche Schreckbewegung, durch welche nach Angabe der Mutter das Gewächs gerade entstanden sein soll, da die Mutter mit je einer Hand die entsprechende Hinterbacke umfaßte, jederseits ein gleiches Gebilde sich entwickelt haben. Unwahrscheinlich ist es, daß durch adhäsive Vorgänge der Tumor seinen Ursprung genommen haben soll; denn dafür spräche kaum eine geringe Fruchtwassermenge, wie sie bei der Geburt des Kindes vorhanden gewesen sein soll, dagegen ist aber die ganze Form des Tumors anzuführen, der mit breiter Basis, fast ohne jede Abgrenzung sich aus der 
Kreuzsteißbeingegend abhebt. Da bei dem Kinde keine deutliche Fovea sacrococcygea gefunden worden ist, so könnte man vielleicht die oben beschriebene feine Öffnung unterhalb des schwanzartigen Anhanges als diese ansehen. Dann könnte man sich vorstellen, daß der Tumor zuerst median sich entwickelt hat. Hiergegen spricht allerdings, daß das die Öffnung umgebende Gewebe absolut keine Verbindung mit fibrösen Strängen hatte, die man als Ligamentum caudale ansehen könnte, welches ,für die Bildung der Fovea coccygea in erster Linie heranzuziehen ist", wie Unger und Brugsch in ihrer Schrift: ,Zur Kenntnis der Fovea und Fistula sacrococcygea s. caudalis und der Entwicklung des Ligamentum caudale beim Menschen"66) nachgewiesen haben.

Überblicken wir alle von uns angeführten Fälle, so erkennen wir, daß die angeborenen Lipo-Fibrome an jeder Stelle der Kreuzsteißbeingegend vorkommen können. Man ist daher wegen des Sitzes eines solchen Tumors in der Fortsetzung des Steißbeines noch nicht berechtigt, diesen als weichen Schwanz zu bezeichnen. Hierzu wäre notwendig, daß man in der Geschwulst zum wenigsten einen Zentralstrang nachweisen. könnte. Andererseits glauben wir aber für das Wesen eines weichen Schwanzes, wie B artels es bei der Beschreibung seines oben, S. 112, wiedergegebenen Falles getan hat, nicht die ,kongenitale Natur" desselben fordern zu müssen, wenn man nicht hierunter die kongenitale Anlage des Tumors, sondern das Vorhandensein desselben bei der Geburt versteht; denn es können, wie Unger und Brug s e h in ihrer Schrift, S. 212, im Sinne von Wiedersh eim hervorheben, solche Körperstellen, wo Organe sich im Stadium der Involution, der phylogenetischen Rückentwicklung befinden, leicht zu Erkrankungen und Neubildungen führen.

\section{Literatur.}

Braun e, W., Die Doppelbildungen und angeborenen Geschwülste der Kreuz* beingegend in anatomischer und klinischer Beziehung. Leipzig 1862.

Mohnike, O., U̇ber geschwänzte Menschen. Münster 1878.

B a r te ls, M., U̇ber Menschenschwänze. Archiv für Anthropologie Bd. XIII, S. $1-41$.

Ders elbe, Eine schwanzähnliche Neubildung beim Menschen. Dieses Archiv Bd, 83 , S. 189 . 
V i c h ow, R., Uber einen Fall von Hygroma cysticum gIntaeale congenitum. Dieses Archiv Bd. 100, S. 571.

Derselbe, Schwanzbildung beim Menschen. Berliner klin. Wochenschr. 1884, Nr. 47, S. 746.

Stolper, P., Die angeborenen Geschwülste der Kreuzsteißbeingegend. Deutsche Zeitschr. für Chir., 50. Bd., H. 3 u. 4, S. 207-280.

Unger, E., und Brugseh, Th., Zur Kenntnis der Forea und Fistula sacrococcygea s. caudalis und der Entwicklung des Ligamentum caudale beim Menschen. Archiv für mikroskop. Anatomie und Entwicklungsgeschichte Bd. 61, 1903, S. 151-219.

\title{
IV. \\ Beitrag zur Kenntnis der Russellschen Körperchen.
}

\author{
Von \\ Johannes Fick,
}

Wien.

Die Annahme, daß die $\mathrm{R}$ u s s ell schen Körperchen (hyaline Kugeln, fuchsinophile Körperchen oder Granulationen) in den Plasmazellen entstehen, ist, wie die folgende historische Übersicht zeigt, schon recht alten Datums. Noch nicht festgestellt ist es aber, ob die Plasmazellen als der einzige Entstehungsort der $\mathrm{R} u \mathrm{~s}$ s e 11 schen Körperchen anzusprechen seien, oder ob auch in andern Zellen, Endothelien, Mastzellen, Leukocyten die Bildung von R u s s ell schen Körperchen vor sich geht. Zur Beantwortung dieser Frage soll die vorliegende Mitteilung einen Beitrag liefern.

Notwendig erscheint es mir zunächst, anzugeben, was ich im folgenden unter der Bezeichnung $\mathrm{Rk}^{\mathbf{1}}$ ) verstehe, da, wie wohl jetzt zweifellos feststeht, diese Bezeichnung nicht von allen Autoren in ein und demselben Sinne gebraucht worden ist. Wenn ich die Bezeichnung Rk den synonymen Benennungen vorziehe, so geschieht dies, weil diese Bezeichnung bezüglich der Natur der fraglichen Gebilde nichts präjudiziert. Ich verstehe nun im folgenden unter Rk jene bekannten, in der Regel kugeligen, manchmal ovoiden, hie und da auch etwas unregelmäßig gestalteten Gebilde, welche sich durch ihren starken Glanz, ihre scharfe Konturierung, ihre

1) $R k=R$ u s s ell sches Körperchen. 